\title{
Weak Convergence of a Random Walk in a Random Environment
}

\author{
Gregory F. Lawler
}

Department of Mathematics, Duke University, Durham, NC 27706, USA

\begin{abstract}
Let $\pi_{i}(x), \quad i=1, \ldots, d, \quad x \in Z^{d}, \quad$ satisfy $\quad \pi_{i}(x) \geqq \alpha>0$, and $\pi_{1}(x)+\ldots+\pi_{d}(x)=1$. Define a Markov chain on $Z^{d}$ by specifying that a particle at $x$ takes a jump of +1 in the $i^{\text {th }}$ direction with probability $\frac{1}{2} \pi_{i}(x)$ and a jump of -1 in the $i^{\text {th }}$ direction with probability $\frac{1}{2} \pi_{i}(x)$. If the $\pi_{i}(x)$ are chosen from a stationary, ergodic distribution, then for almost all $\pi$ the corresponding chain converges weakly to a Brownian motion.
\end{abstract}

\section{Introduction}

Let $Z^{d}$ be the integer lattice and let $e_{i}, i=1, \ldots, d$, denote the unit vector whose $i^{\text {th }}$ component is equal to 1 . Let

$$
S=\left\{\left(p_{1}, \ldots, p_{d}\right) \in \mathbb{R}^{d}: p_{i} \geqq 0, p_{1}+\ldots+p_{d}=1\right\},
$$

and suppose we have a function $\pi: Z^{d} \rightarrow S$. Then a Markov chain $X_{\pi}(j)$ on $Z^{d}$ is generated with transition probability

$$
P\left\{X_{\pi}(j+1)=x \pm e_{i} \mid X_{\pi}(j)=x\right\}=\frac{1}{2} \pi_{i}(x),
$$

and generator

$$
L_{\pi} g(x)=\sum_{i=1}^{d} \frac{1}{2} \pi_{i}(x)\left\{g\left(x+e_{i}\right)+g\left(x-e_{i}\right)\right\}
$$

If the function $\pi$ is chosen from some probability distribution on $S$, this gives an example of a random walk in a random environment.

For any $\pi$, we can consider the limiting distribution of the process $X_{\pi}$ satisfying $X_{\pi}(0)=0$ and (1.1). Let $\alpha>0$ and set

$$
S^{\alpha}=\left\{\left(p_{1}, \ldots, p_{d}\right) \in S: p_{i} \geqq \alpha\right\},
$$

and let $C^{\alpha}$ be the set of functions $\pi: Z^{d} \rightarrow S^{\alpha}$. The main result of this paper is:

Theorem 1. Let $\mu$ be a stationary ergodic measure on $C^{\alpha}$. Then there exists $b \in S^{\alpha}$ such 
that for $\mu$-almost all $\pi \in C^{\alpha}$, the processes

$$
X_{\pi}^{(n)}(t)=\frac{1}{\sqrt{n}} X_{\pi}([n t])
$$

converge in distribution to a Brownian motion with covariance $\left(b_{i} \delta_{i j}\right)$

A special case of this theorem occurs when the $\pi(x)$ are independent, identically distributed random variables taking values in $S^{\alpha}$.

A similar theorem for diffusion processes with random coefficients was proved by Papanicolaou and Varadhan [3], and a considerable portion of this paper is only a restating of their proof in the context of discrete random walk. The crucial new step is Lemma 4, which replaces Lemma 3.1 of their paper. This is a discrete version of an a priori estimate for solutions of uniformly elliptic equations. The ideas of Krylov [2] are used in the proof of Lemma 4; properties of concave functions are used to estimate solutions to a discrete Monge-Ampere equation.

\section{An Ergodic Theorem on the Space of Environments}

Fix an environment $\pi \in C^{\alpha}$, and assume $X_{n}(0)=0$. Let $Z_{j}=\left(Z_{j}^{1}, \ldots, Z_{j}^{d}\right)=$ $X_{\pi}(j)-X_{\pi}(j-1)$, and let $\mathscr{T}_{j}=\sigma\left\{Z_{1}, \ldots, Z_{j}\right\}$. Let $Y_{j}=\pi\left(X_{n}(j)\right)$. Then $Y_{j}$ is measurable with respect to $\mathscr{T}_{j}$, and

$$
P\left\{Z_{j}=e_{i} \mid \mathscr{T}_{j-1}\right\}=P\left\{Z_{j}=-e_{i} \mid \mathscr{T}_{j-1}\right\}=\frac{1}{2} Y_{j}^{i} .
$$

Then $X_{\pi}(n)=\sum_{j=1}^{n} Z_{j}$ is a martingale and

$$
\mathscr{E}\left(Z_{j}^{i_{1}} Z_{j}^{i_{2}} \mid \mathscr{T}_{j-1}\right)=\left\{\begin{array}{ll}
0 & i_{1} \neq i_{2} \\
Y_{j-1}^{i_{1}} & i_{1}=i_{2}
\end{array} .\right.
$$

Let $V_{n}^{i}=\sum_{j=0}^{n-1} Y_{j}^{i}$. Then the invariance principle for martingales (see e.g. Theorem 4.1 of [1]) states that $W_{n}(t)=\left(W_{n}^{1}(t), \ldots, W_{n}^{d}(t)\right)$ converges in distribution to the standard Brownian motion on $\mathbb{R}^{d}$, where

$$
W_{n}^{i}(t) \equiv\left(V_{n}^{i}\right)^{-1 / 2} \sum_{j=1}^{[n t]} Z_{j}^{i} .
$$

Now suppose there exists a $b \in S^{\alpha}$ such that

$$
\lim _{n \rightarrow \infty} \frac{1}{n} \sum_{j=0}^{n-1} \pi\left(X_{n}(j)\right)=b \text { a.s. }
$$

Then by the above argument we can conclude that

$$
X^{(n)}(t)=\frac{1}{\sqrt{n}} X_{\pi}([n t])
$$

converges in distribution to a Brownian motion with covariance $\left(b_{i} \delta_{i j}\right)$. Therefore, in order to prove Theorem 1 it is sufficient to prove: 
Theorem 2. Let $\mu$ be a stationary ergodic probability measure on $C^{\alpha}$. Then there exists $b \in S^{\alpha}$ such that for $\mu$-almost all $\pi \in C^{\alpha}$,

$$
\lim _{n \rightarrow \infty} \frac{1}{n} \sum_{j=0}^{n-1} \pi\left(X_{\pi}(j)\right)=b \text { a.s. . }
$$

This is clearly an ergodic theorem and the idea of Papanicolaou and Varadhan [3] is to find a measure on $C^{\alpha}$ so that a standard ergodic argument can be used.

We define the canonical Markov chain with state space $C^{\alpha}$ to be the chain whose generator $\mathscr{L}$ is given by

$$
\mathscr{L} g(\pi)=\sum_{i=1}^{d} \frac{1}{2} \pi_{i}(0)\left\{g\left(\tau_{e_{i}} \pi\right)+g\left(\tau_{-e_{i}} \pi\right)\right\},
$$

where $\tau_{x} \pi(y)=\pi(y-x)$. In this chain, the "particle" stays fixed at the origin and allow the environment to change around it (rather than having the particle move around a fixed environment). If we define $g_{0}: C^{\alpha} \rightarrow \mathbb{R}^{d}$ by $g_{0}(\pi)=\pi(0)$, and let $\mathscr{L}^{j} \pi$ denote the (random) environment at the $j^{\text {th }}$ step of this chain, then (2.1) is equivalent to

$$
\lim _{n \rightarrow \infty} \frac{1}{n} \sum_{j=0}^{n-1} g_{0}\left(\mathscr{L}^{j} \pi\right)=b \text { a.s. } \mu .
$$

By standard ergodic theory we can prove (2.2), and hence (2.1), if we prove:

Theorem 3. Let $\mu$ be a stationary ergodic probability measure on $C^{\alpha}$. Then there exists an ergodic probability measure $\lambda$ on $C^{\alpha}$ which is mutually absolutely continuous with $\mu$ and which is invariant under the canonical Markov chain $\mathscr{L}$.

Clearly,

$$
b=\int_{C^{\alpha}} g_{0}(\pi) d \lambda(\pi)
$$

To prove Theorem 3 we need some lemmas. For each $n>0$, let $T_{n}$ denote the elements of $Z^{d}$ under the equivalence relation

$$
\left(z_{1}, \ldots, z_{d}\right) \sim\left(w_{1}, \ldots, w_{d}\right) \text { if } \frac{1}{2 n}\left(z_{i}-w_{i}\right) \in Z \text { for each } i .
$$

Then $\left|T_{n}\right|=(2 n)^{d}$. If $\pi: T_{n} \rightarrow S^{\alpha}$, we may think of $\pi$ as a periodic environment in $C^{\alpha}$. Let $C_{n}^{\alpha}$ denote the set of such periodic environments. For $\pi \in C_{n}^{\alpha}$, let $R_{\pi}^{n}$ denote the resolvent operator

$$
R_{\pi}^{n} g(x)=\sum_{j=0}^{\infty}\left(1-\frac{1}{n^{2}}\right)^{j} L_{\pi}^{j} g(x) .
$$

If $g: T_{n} \rightarrow \mathbb{R}$ we define the usual $L^{p}$ norms (with respect to normalized counting measure on $T_{n}$ ),

$$
\begin{aligned}
\|g\|_{p} & =\left|(2 n)^{-d} \sum_{x \in T_{n}}(g(x))^{p}\right|^{1 / p} \\
\|g\|_{\infty} & =\sup _{x \in T_{n}}|g(x)|
\end{aligned}
$$


Lemma 4. There exists a constant $c_{1}$ (depending only ond and $\alpha$ ) such that for every $\pi \in C_{n}^{\alpha}, g: T_{n} \rightarrow R$,

$$
\left\|R^{n} g\right\|_{\infty} \leqq c_{1} n^{2}\|g\|_{d} .
$$

The proof of this lemma is delayed until Sect. 3. The next lemma follows from our assumption that $\mu$ is stationary (see Parthasarathy [4]).

Lemma 5. For each $n$, there exists $\pi_{n} \in C_{n}^{\alpha}$ such that if $\mu_{n}$ is the probability measure on $C^{\alpha}$ which assigns measure $(2 n)^{-d}$ to $\tau_{x} \pi_{n}$ for each $x \in T_{n}$, then

$$
\mu_{n} \rightarrow \mu \text { weakly. }
$$

Proof of Theorem 3. Let $\pi_{n} \in C_{n}^{\alpha}$ be a sequence as in Lemma 5 with $\mu_{n} \rightarrow \mu$. Let $\phi_{n}$ be the density, with respect to normalized counting measure on $T_{n}$, of an invariant probability measure on $T_{n}$ for $\pi_{n}$, i.e. $L_{\pi_{n}} \phi_{n}=\phi_{n}$ and $\left\|\phi_{n}\right\|_{1}=1$. If $R_{n}=R_{\pi_{n}}^{n}$ is the resolvent corresponding to $\pi_{n}$, then $R_{n} \phi_{n}=n^{2} \phi_{n}$. If we consider $R_{n}$ as a map from $L^{d}\left(T_{n}\right)$ to $L^{\infty}\left(T_{n}\right)$, then Lemma 4 states that the map is bounded by $c_{1} n^{2}$. Therefore $R_{n}^{*}: L^{1}\left(T_{n}\right) \rightarrow L^{d /(d-1)}\left(T_{n}\right)$ is also bounded by $c_{1} n^{2}$. Since $R_{n}^{*} \phi_{n}=n^{2} \phi_{n}$, we get

$$
\begin{gathered}
n^{2}\left\|\phi_{n}\right\|_{d /(d-1)} \leqq c_{1} n^{2}\left\|\phi_{n}\right\|_{1}=c_{1} n^{2} \\
\left\|\phi_{n}\right\|_{a /(d-1)} \leqq c_{1} .
\end{gathered}
$$

Let $\lambda_{n}$ be the probability measure on $C_{n}^{\alpha}$,

$$
\lambda_{n}\left(\tau_{x} \pi_{n}\right)=(2 n)^{-d} \phi_{n}(x)
$$

Then $\lambda_{n}$ is invariant under the canonical Markov chain $\mathscr{L}$ and

$$
\left\|\frac{d \lambda_{n}}{d \mu_{n}}\right\|_{d /(d-1)} \leqq c_{1} .
$$

Since $\mu_{n} \rightarrow \mu$ weakly, standard arguments give that $\lambda_{n}$ has a subsequence converging to a probability measure $\lambda$ which is invariant under $\mathscr{L}$. Also $\lambda \ll \mu$ and, in fact,

$$
\int_{C^{\alpha}}\left|\frac{d \lambda}{d \mu}\right|^{d /(d-1)} d \mu \leqq c_{1}^{d /(d-1)} .
$$

Let $E=\{d \lambda / d \mu=0\}$. Since $\lambda$ is invariant, $\lambda(\mathscr{L} E)=\lambda(E)=0$, and hence $\mathscr{L} E \subset E$ (a.s. $\mu$ ). Since $\mu$ is ergodic and $\lambda \ll \mu, \mu(E)=0$, and hence $\mu \ll \lambda$. Since $\mu$ and $\lambda$ are mutually absolutely continuous and $\mu$ is ergodic, $\lambda$ is ergodic.

Example. Let $d=2$ and $\mu$ be product measure with $\mu\{\pi(x)=\alpha\}=\mu\{\pi(x)=1-\alpha\}=\frac{1}{2}$, where $0<\alpha<\frac{1}{2}$. Then $\mu$ is not invariant under $\mathscr{L}$, if $B=\left\{\pi\left(e_{1}\right)=\alpha\right\}$, then $\mu(B)=\frac{1}{2}$, but $\mu(\mathscr{L} B)=\frac{3}{8}+\frac{\alpha}{4}$. Although it is not easy to describe $\lambda$ in this case, symmetry considerations give that $b=\left(\frac{1}{2}, \frac{1}{2}\right)$. 


\section{Proof of Lemma 4}

It remains to prove Lemma 4. Let

$$
\begin{aligned}
D_{n} & =\left\{\left(z_{1}, \ldots, z_{d}\right) \in Z^{d}:\left|z_{1}\right|+\ldots+\left|z_{d}\right| \leqq n\right\}, \\
\partial D_{n} & =\left\{z \in D_{n}:\left|z_{1}\right|+\ldots+\left|z_{d}\right|=n\right\}, \\
\text { int } D_{n} & =D_{n} / \partial D_{n} .
\end{aligned}
$$

Let $\pi \in C_{n}^{\alpha}$. If $f: D_{n} \rightarrow[0, \infty)$ with $f(x)=0$ for $x \in \partial D_{n}$, let

$$
Q f(x)=E_{x} \sum_{j=0}^{\tau} f\left(X_{\pi}(j)\right),
$$

where $\tau=\inf \left\{j: X_{n}(j) \in \partial D_{n}\right\}$, and $E_{x}$ denotes expectation assuming $X_{n}(0)=x$. We will prove the following:

Lemma 6. There exists a constant $c_{2}$ (depending only on $d$ and $\alpha$ ) such that for every $f: D_{n} \rightarrow[0, \infty)$,

$$
\|Q f\|_{\infty} \leqq c_{2} n^{2}\|f\|_{d}
$$

where

$$
\|f\|_{d}^{d}=\frac{1}{\left|D_{n}\right|} \sum_{x \in D_{n}}(f(x))^{d} .
$$

To get Lemma 4 from Lemma 6 is routine using the fact that the expected time until hitting $\partial D_{n}$ is of order $n^{2}$.

Fix $n$, and write $D=D_{n}$. If $u: D \rightarrow \mathbb{R}$, we define the second difference operators on int $D$ by

$$
\Delta_{i} u(x)=u\left(x+e_{i}\right)+u\left(x-e_{i}\right)-2 u(x) .
$$

We will call $u$ concave on $D$ if $\Delta_{i} u(x) \leqq 0$ for all $x \in \operatorname{int} D$ and all $i$ (note this is weaker than the usual definition of concave). We define the discrete Monge-Ampere operator $M$ on int $D$ by

$$
M u=\prod_{i=1}^{d} \Delta_{i} u .
$$

we will prove the following:

Lemma 7. Let $f: D \rightarrow[0, \infty)$ be a function with $f \equiv 0$ on $\partial D$. Then there exists a concave function $z: D \rightarrow[0, \infty)$ such that

(i) $z \equiv 0$ on $\partial D$,

(ii) $(-1)^{d} M z=f^{d}$ on int $D$.

Moreover, there exists a constant $c_{3}$ (depending only on d) such that

(iii) $\|z\|_{\infty} \leqq c_{3} n^{2}\|f\|_{d}$.

Suppose that we have Lemma 7, and let us derive Lemma 6. Fix $x \in \operatorname{int} D$, and let 
$X_{\pi}(j)$ be the Markov chain induced by $\pi$ with $X_{\pi}(0)=x$. Then

$$
\begin{aligned}
E\left(z\left(X_{\pi}(1)\right)-z\left(X_{\pi}(0)\right)\right. & =\sum_{i=1}^{d} \frac{1}{2} \pi_{i}(x) \Delta_{i} z(x) \\
& \leqq-\frac{1}{2} \alpha|M z(x)|^{1 / d} \\
& =-\frac{1}{2} \alpha f(x) .
\end{aligned}
$$

Here we have used the inequality $\left(a_{1} b_{1}+\ldots+a_{d} b_{d}\right)^{d} \geqq\left(a_{1} \ldots a_{d}\right) \quad\left(b_{1} \ldots b_{d}\right)$. Continuing as above we may deduce

$$
E\left[z\left(X_{\pi}(j \wedge \tau)\right)-z\left(X_{\pi}(0)\right)+\frac{1}{2} \alpha \sum_{k=0}^{(j-1) \wedge \tau} f\left(X_{\pi}(k)\right)\right] \leqq 0 .
$$

Letting $j$ go to infinity,

$$
\frac{1}{2} \alpha Q f(x)=E_{x} \frac{1}{2} \alpha \sum_{k=0}^{\tau} f\left(X_{\pi}(k)\right) \leqq z(x) .
$$

and Lemma 7 then gives the required bound.

To prove Lemma 7, let $\mathscr{A}$ be the set of all concave functions $u$ on $D$ satisfying

(i) $u \equiv 0$ on $\partial D$,

(ii) $(-1)^{d} M u \geqq f^{d}$ on int $D$.

We first note that $\mathscr{A}$ is non-empty: let $h: D \rightarrow[0, \infty)$ by

$$
h(x)=n(n+1)-|x|(|x|+1),
$$

where $\left|\left(x_{1}, \ldots, x_{d}\right)\right|=\left|x_{1}\right|+\ldots+\left|x_{d}\right|$. One can check that $(-1)^{d} M h \geqq 2^{d}$ and hence $\beta h \in \mathscr{A}$ for $\beta$ sufficiently large.

It is easy to check that if $u_{1}, u_{2} \in \mathscr{A}$, then $\min \left(u_{1}, u_{2}\right) \in \mathscr{A}$; in fact, if we let

$$
z(x)=\inf _{u \in \mathscr{A}} u(x)
$$

one can verify that $z \in \mathscr{A}$. It remains to be shown that $(-1)^{d} M z=f^{d}$. Suppose $(-1)^{d} M z(x)>(f(x))^{d}$ for some $x \in$ int $D$, i.e.

$$
(-1)^{d} \prod_{i=1}^{d}\left(z\left(x+e_{i}\right)+z\left(x-e_{i}\right)-2 z(x)\right)>(f(x))^{d} .
$$

Let $\gamma<z(x)$ be such that

and set

$$
(-1)^{d} \prod_{i=1}^{d}\left(z\left(x+e_{i}\right)+z\left(x-e_{i}\right)-2 \gamma\right)=(f(x))^{d} .
$$

$$
v(y)=\left\{\begin{array}{ll}
z(y) & y \neq x \\
\gamma & y=x
\end{array} .\right.
$$

Then again one can check that $v \in \mathscr{A}$, contradicting the minimality of $z$.

We now wish to estimate $z$. For $x \in$ int $D$, let

$$
\begin{aligned}
I(x)=\left\{\left(a_{1}, \ldots, a_{d}\right)\right. & \in R^{d}: z\left(x+e_{i}\right)-z(x) \\
& \left.\leqq a_{i} \leqq z(x)-z\left(x-e_{i}\right)\right\} .
\end{aligned}
$$


Note that meas $(I(x))=(-1)^{d} M z(x)=(f(x))^{d}$. We state the next easily provable fact as a lemma:

Lemma 8. Let $a \in R^{d}, b>0$, and let $r$ be the affine function $r(x)=a \cdot x+b$. Suppose $r(x) \geqq z(x)$ for every $x \in D$ and $r\left(x_{0}\right)=z\left(x_{0}\right)$ for some $x_{0} \in \operatorname{int} D$. Then $a \in I\left(x_{0}\right)$.

Now let $\bar{z}=\|z\|_{\infty}$ and let $\bar{x} \in \operatorname{int} D$ with $z(\bar{x})=\bar{z}$. Assume $\bar{z}>0$. Let

$$
A=\left\{a \in \mathbb{R}^{d}:|a| \leqq \vec{z} / 4 n\right\} \text {. }
$$

Fix $a \in A$. If $b \geqq \frac{3}{2} \bar{z}$, then $a \cdot x+b>\bar{z} \geqq z(x)$ for every $x \in D$. Therefore there exists a least $b$ (depending on a) such that $a \cdot x+b \geqq z(x)$ for all $x \in D$. It is easy to see that $a \cdot x_{0}+b=z\left(x_{0}\right)$ for some $x_{0} \in D$, and since

$$
a \cdot x_{0}+b=a \cdot \bar{x}+b+a \cdot\left(x_{0}-\bar{x}\right) \geqq \frac{1}{2} \bar{z}>0,
$$

$x_{0} \in$ int $D$. By Lemma $8, a \in I\left(x_{0}\right)$. Therefore

$$
\begin{aligned}
A & \subset \bigcup_{x \in \operatorname{int} D} I(x), \\
\operatorname{meas}(A) & \leqq \operatorname{meas}(\bigcup I(x)), \\
& \leqq \sum_{x \in D}\left((f(x))^{d}\right.
\end{aligned}
$$

Since meas $(A)=\left(\bar{z}^{d}\right)\left(c_{4} n\right)^{-d}$ for some $c_{4}$, we get

$$
\begin{aligned}
\bar{z} & \leqq c_{4} n\left[\sum_{x \in D}(f(x))^{d}\right]^{1 / d} \\
& \leqq c_{3} n^{2}\|f\|_{d} .
\end{aligned}
$$

Acknowledgements. I would like to thank S. R. S. Varadhan for suggesting this problem and for bringing to my attention the work of Krylov. I would also like to thank Bob Vanderbei for useful discussions.

This paper was written while the author was a visiting member at the Courant Institute of Mathematical Sciences.

\section{References}

1. Hall, P., Heyde, C. C.: Martingale limit theory and its application. New York: Academic Press 1980

2. Krylov, N. V.: An inequality in the theory of stochastic integrals. Theor. Prob. Appl. 16, 438-448 (1971)

3. Papanicolaou, C., Varadhan, S. R. S.: Diffusions with Random coefficients. In: Essays in Honor of C. R. Rao. Amsterdam: North Holland 1982

4. Parthasarathy, K. R.: On the category of ergodic measure. Ill. J. Math. 5, 648-655 (1961)

Communicated by T. Spencer

Received May 20, 1982 
